\title{
Editorial: Limits on Special Issues
}

\author{
Philip Machanick sacj.editor@gmail.com \\ Department of Computer Science, Rhodes University, South Africa
}

\section{Introduction}

At the end of 2018, I announced that James Dibley was leaving us as Production Editor. He has found a way to continue - welcome back. I would also like to thank Marie Hattingh for taking on the role of invoicing for publication charges, relieving me of a burden.

A few minor changes are in the offing: we need to start reflecting ORCID (Open Researcher and Contributor ID) iDs in the PDF version of our papers as well as in the web metadata, to meet requirements of SciELO (Scientific Electronic Library Online), an online open-access portal, that originated in Brazil ${ }^{1}$. Membership of the South African version ${ }^{2}$ is one of the criteria for accreditation as a subsidy-earning publication by the South African Department of Higher Education and Training (DHET). SACJ was invited to join the SciELO South Africa site in 2018 and is now accredited for two reasons: membership of SciELO and inclusion in the Scopus index. Previously, we were accredited according to DHET's South Africa-specific list.

Why "ORCID" and "iD" when the "ID" in the main name stands for identifier? The latter appears in the logo and - absent any clear explanation - appears to be a consequence of graphic design.

I reported on SciELO in more detail in my last editorial.

Why are ORCID iDs useful? They help to disambiguate common author names and are increasingly being adopted by funding agencies like the South African National Research Foundation (NRF). Expect in future issues to have author names appear in a format like this (this does not exactly conform to the specification ${ }^{3}$; we are working on it) - the ORCID iD logo is clickable and takes you to the author's page: Philip Machanick (iD) .

In this editorial, I focus on where we are going with special issues because Scopus listing has made us more visible and hence a target of requests for special issues.

Machanick, P. (2019). Editorial: Limits on Special Issues [Editorial]. South African Computer Journal 31(1), viiviii. https://doi.org/10.18489/sacj.v31i1.727

Copyright (C) the author(s); published under a Creative Commons NonCommercial 4.0 License (CC BY-NC 4.0).

$S A C J$ is a publication of the South African Institute of Computer Scientists and Information Technologists. ISSN 1015-7999 (print) ISSN 2313-7835 (online).

${ }^{1}$ https://www.eldis.org/organisation/A6815

${ }^{2}$ http://www.scielo.org.za/

${ }^{3} \mathrm{https}$ ///orcid.org/content/journal-article-display-guidelines; the version used here is based on this example: https://tex.stackexchange.com/questions/498688/orcid-in-latex-file-of-ieee-trans-in-a-customized-position 


\section{Special Issues}

In 2017, we tried an experiment with the Health Informatics South Africa (HISA) conference in which authors were invited to submit a paper that could be accepted in the journal up front, rather than an extended paper after the event. I thank guest editor Nicky Mostert of Nelson Mandela University for undertaking this experiment. Unfortunately conference deadlines and journal quality did not work out well and only one paper was ultimately accepted as a result.

Since then we have done a rethink on special issues. Our inclusion in Scopus has resulted in numerous requests, most of which we have to turn down, for conference-based issues because we do not have the capacity to handle them.

One of our biggest limiting factors is reviewers. If we take on a special issue of a conference with a large number of papers, our existing reviewer base will be overwhelmed. If we rely on a guest editor to supply reviewers, we have no control over quality. Consequently, we are limiting special issues to those most coherently motivated, or those from sources we know (e.g., extended papers from local conferences).

If you want to do a special issue, by all means motivate it-but we are not accepting most motivations unless they are very well organized and clear on their goals.

\section{In this issue}

In this issue, we have 4 research papers:

- Chiridza et al.: "A smart home environment to support risk monitoring for the elderly living independently"4

- Le Roux et al.: "Parsing and analysis of a Xilinx FPGA bitstream for generating new hardware by direct bit manipulation in real-time"

- Mujinga et al.: "Towards a framework for online information security applications development: A socio-technical approach"

- Parry: "Computing research in South Africa: A scientometric investigation"

\section{Future}

An issue the editorial team is debating is using the Internet Archive, also known as the Wayback Machine, for URL references. You can ask the archive to create a copy of any web page that allows web crawlers ${ }^{5}$ to make an archival version as it was on that specific date. This works around the problem that web references can be ephemeral. On the whole, we prefer refereed academic sources that have a DOI, thereby ensuring not only independent review but also greater permanence.

\footnotetext{
${ }^{4}$ This paper was submitted as an extended paper for the HISA 2017 conference.

${ }^{5}$ https://archive.org/web
} 